\title{
Papel de las quimiocinas en la inmunopatogénesis del linfoma cutáneo de células T
}

Role of chemokines in the immunopathogenesis of cutaneous T cell lymphoma

\section{María Natalia Mejía1, Margarita María Velásquez ${ }^{2}$}

1. Médica, residente de segundo año de Dermatología, Sección de Dermatología, Facultad de Medicina, Universidad de Antioquia, Medellín, Colombia

2. Médica dermatóloga, doctora en Ciencias Básicas Biomédicas con énfasis en Inmunología; profesora, Sección de Dermatología, Facultad de Medicina, Universidad de Antioquia; Centro de Investigaciones Dermatológicas, CIDERM, Medellín, Colombia

\section{Resumen}

Los linfomas cutáneos de células T representan una expansión maligna de linfocitos de memoria que muestran una gran predilección por la piel. Son enfermedades de curso largo e indolente que pueden afectar la calidad de vida de forma significativa.

Las quimiocinas son una gran familia de citocinas relacionadas con la quimiotaxis, supervivencia y localización celular. Actúan sobre sus células blanco mediante receptores acoplados a proteínas G. En esta revisión se trata el papel que juegan las quimiocinas en el asentamiento de las células tumorales en la piel y su posterior relación con la infiltración de otros órganos; además; sobre cómo ellas son un posible blanco terapéutico para el manejo de estas enfermedades.

PALABRAS ClAve: linfoma cutáneo de células T, quimiocinas, CCL17, CCL22, CCR4, CXCR4.

\section{Summary}

The cutaneous T-cell lymphoma represents an expansion of malignant memory $\mathrm{T}$ cells that presents important predominance for the skin. These are diseases of long and indolent course, but that may affect significantly the quality of life. Chemokines are a large family of cytokines related to chemotaxis, survival and cellular homing. They act on their target cells via $\mathrm{G}$ protein-coupled receptors. This review will address the role of chemokines in the homing of tumor cells in the skin, and their subsequent relationship with infiltration of other organs, and also as they are a potential therapeutic target for the management of these diseases

KEY WORDS: cutaneous T cell lymphoma, chemokines, CCL17, CCL22, CCR4, CXCR4

\section{Correspondencia:}

Margarita Velásquez

Email:

mmvelasquez@yahoo.com

Recibido: 10 de marzo de 2013.

Aceptado: 13 de junio de 2013.

No se reportan conflictos de intereses.

\section{Introducción}

Las quimiocinas son una gran familia de citocinas multifuncionales quimiotácticas, que se agrupan en cuatro familias con base en el espacio de los residuos de cis-

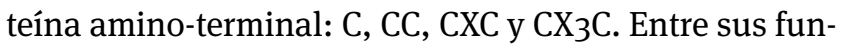
ciones se encuentran la quimiotaxis, la supervivencia de algunas células del sistema inmunológico y su localización con los antígenos ${ }^{1}$.

Las quimiocinas se unen a sus células blanco mediante receptores acoplados a proteínas $\mathrm{G}$, que se expresan de forma diferencial según el estado de diferenciación o activación celular ${ }^{2,3}$.

El linfoma cutáneo primario es un grupo heterogéneo 
de linfomas T y B que tienen diferentes inmunofenotipos, presentaciones clínicas y pronóstico. Son el segundo grupo de linfomas no Hodgkin extraganglionares más comunes después de los gastrointestinales ${ }^{4}$. Se clasifican, según la Organización Mundial de la Salud (OMS), en linfomas de células T y NK (Natural Killer), linfomas B y linfomas de precursores hematopoyéticos ${ }^{5}$. Las formas más frecuentes de los linfomas cutáneos de células T (Cutaneous T-cell Lymphoma) son la micosis fungoide y el síndrome de Sèzary. Los linfomas cutáneos de células $\mathrm{T}$ representan una expansión maligna de linfocitos de memoria $\mathrm{CD} 4+\mathrm{CD} 45 \mathrm{RO}+$, cuyo órgano blanco primario es la piel ${ }^{6}$.

La micosis fungoide se caracteriza por un curso clínico lento que progresa a través de diferentes estadios, con parches, placas hasta la fase tumoral, y con compromiso de otros órganos y sistemas7. El síndrome de Sèzary es una variante leucémica ${ }^{8}$ que se caracteriza por la presencia de eritrodermia, linfadenopatías y más de 1.000 células malignas con núcleo cerebriforme en sangre periférica, llamadas células de Sèzary ${ }^{9,10}$.

El mecanismo responsable de la localización en la piel de estas células no está completamente entendido, y las quimiocinas podrían ser las responsables del asentamiento del clon maligno de linfocitos T en la piel.

Esta revisión está enfocada en las quimiocinas y los receptores de quimiocinas, de los que se ha dicho que juegan un papel en la inmunopatogénesis del linfoma cutáneo de células $\mathrm{T}$.

\section{Epidermotropismo favorecido por CXCR3 y sus ligandos CXCL9 y CXCL10}

Las quimiocinas CXCL9, CXCL10 y CXCL11 se han relacionado con la quimiotaxis de células Th1 a los sitios de inflamación; ambas son inducidas por las citocinas de perfil Th1 y el interferón gamma (IFN-g) ${ }^{1,11}$; estas quimiocinas se unen al receptor CXCR3.

Di Lu, et al., tomaron biopsias de piel en pacientes con micosis fungoide en diferentes estadios, midieron la expresión de CXCR3 en ellas, tanto en linfocitos reactivos como en el infiltrado maligno, y demostraron que el receptor CXCR3 se encuentra en las células linfoides tumorales en casos de micosis fungoide en estadios iniciales y no en otras células epidérmicas; además, en fases avanzadas de la enfermedad, la expresión se CXCR3 se pierde. Posteriormente, Kallinich, et al., obtuvieron hallazgos similares con un modelo experimental semejante ${ }^{12,13}$.

En estudios que buscaban dilucidar el mecanismo del epidermotropismo del clon maligno, se hicieron mediciones de diferentes quimiocinas. Por medio de un antisuero CXCL10, se midió su expresión en la piel de pacientes con micosis fungoide y controles sanos, $\mathrm{y}$ se demostró que normalmente dicha quimiocina solo se encuentra en los queratinocitos basales, pero, en la micosis fungoide la expresión de CXCL1o está aumentada en los queratinocitos basales y suprabasales, y se encuentra aún en mayores concentraciones alrededor del infiltrado linfoide maligno ${ }^{14}$; la quimiocina CXCL9 se expresa simultáneamente con CXCL10, pero en menor cantidad ${ }^{15}$.

Esto sugiere que el receptor CXCR3 está involucrado en el epidermotropismo de las células tumorales que se encuentra en las fases iniciales de la micosis fungoide.

\section{Migración transendotelial y gravedad de la enfermedad por CCR4 y sus ligandos CCL17 y CCL22}

En diferentes estudios se ha demostrado en biopsias de piel con micosis fungoide comparadas con biopsias de controles sanos, que las células tumorales transformadas en la micosis fungoide tienen una mayor expresión de CCR4 y responden a sus ligandos CCL22 y CCL17; además, dichas quimiocinas aumentaron la expresión de integrinas en las células tumorales y la unión de estas a sus ligandos en las células endoteliales, lo que les permite mayor presencia en la piel. Dicho estudio se basó en un modelo derivado de células de pacientes con micosis fungoide o síndrome de Sèzary, y con linfocitos $\mathrm{T}$ sanos como controles. La principal fuente de estas dos quimiocinas en la piel son las células dendríticas y las células endoteliales ${ }^{13,16,17 .}$ La expresión de CCR4 también se evidenció en células de linfoma cutáneo anaplásico de células grandes ${ }^{18}$.

La quimiocina CCL17 se expresa en la piel de los pacientes con micosis fungoide en todas las etapas de la enfermedad, lo cual no sucede controles sanos. Además, los niveles séricos de CCL17 fueron mayores en la micosis fungoide que en controles sanos o con psoriasis vulgar. Las concentraciones de CCL17 se relacionan con la gravedad de la enfermedad, ya que fueron mayores en el estadio tumoral en comparación con los estadios iniciales.

Se han reportado hallazgos similares sobre la expresión de CCL22 en todas las etapas de la enfermedad, pero no se encontró diferencia entre sus diferentes estadios $^{19}$. En los pacientes con síndrome de Sèzary se ha 
reportado que las células malignas circulantes expresan CCR4 en su membrana ${ }^{20}$. La importancia de este receptor de quimiocinas se evidencia con estudios con bexaroteno. Este es un retinoide aprobado por la Food and Drug Administration (FDA), en 2009, para el tratamiento de los linfomas cutáneos de células T. Se ha informado que algunos pacientes con síndrome de Sèzary tratados con este medicamento presentaron una importante mejoría de la eritrodermia; en estudios in vitro se evidenció que el bexaroteno disminuye la expresión de CCR4 y la quimiotaxis en respuesta a la CCL17 ${ }^{21}$.

\section{CCR10 y su ligando CCL27}

La CCL27 es exclusiva y constitutivamente producida en la piel por los queratinocitos epidérmicos. Esta quimiocina se une al receptor CCR10 ${ }^{22,23}$. En pacientes con el síndrome de Sèzary se demostró que las células neoplásicas circulantes tenían una gran expresión de CCR1o al medir la expresión del ARNm para dicho receptor; incluso, la expresión sobre los valores normales es mayor para CCR1o que para otros receptores de quimiocinas ${ }^{24}$. Lo mismo se observa cuando se mide en las biopsias de piel de pacientes con micosis fungoide, síndrome de Sèzary $u$ otros linfomas cutáneos de células $\mathrm{T}^{25}$.

En los estadios iniciales de la micosis fungoide hay mayor número de células $\mathrm{CD} 4+\mathrm{CCR} 10+$ en sangre periférica y en piel. Además, las concentraciones séricas de su ligando CCL27, están elevadas en la micosis fungoide cuando se comparan con controles normales, al igual que en los queratinocitos basales, suprabasales y las células endoteliales de los plexos dérmicos ${ }^{26}$.

Se ha reportado la relación entre las concentraciones séricas de CCL27 y otros marcadores de enfermedad, como el índice de carga tumoral, las lesiones en piel y el número de células de Sèzary circulantes, los cuales disminuyeron luego del tratamiento y la mejoría de las lesiones cutáneas ${ }^{27,28}$. Todos estos hallazgos sugieren que la CCL27 y su receptor CCR1o son importantes en la ubicación de las células tumorales en la piel, en los estadios tempranos de la micosis fungoide o el síndrome de Sèzary.

\section{Migración de los linfocitos T malignos a la piel}

Para tratar de dilucidar cómo se produce la migración de las células tumorales a la piel, Hoeller, et al., hicieron estudios de epifluorescencia intravital en tiempo real y microscopía de dos fotones; demostraron que las células del síndrome de Sèzary ruedan a lo largo de las vénulas dérmicas por un mecanismo dependiente de selectinas, y que las quimiocinas CCL17 y CCL27 son necesarias para la adhesión firme al endotelio y la posterior extravasación a la piel ${ }^{29}$.

\section{Microambiente Th 2: CCR3 y sus ligandos CCL11 y CCL26}

Las quimiocinas CCL11 y CCL26 tienen una importante actividad quimiotáctica para las células $\mathrm{CCR}_{3}+$, como los eosinófilos, los basófilos y las subpoblaciones de células Th2 $2^{2,30-32}$. Como el perfil Th2 disminuye la respuesta antitumoral, varios investigadores han estudiado la expresión de estas quimiocinas en el linfoma cutáneo de células T. Se ha encontrado que en estos pacientes los fibroblastos expresan más CCL26 que los fibroblastos de piel sana, y dicha expresiónes aún mayor en los estadios avanzados de la enfermedad. Las concentraciones de ARNm de dicha quimiocina aumentan en presencia de IL-4.

De la misma manera, las concentraciones séricas de CCL11 y CCL26 están aumentadas en los estadios avanzados del síndrome de Sèzary y la micosis fungoide, al compararlos con controles sanos o las fases iniciales. Las quimiocinas disminuyen si el tratamiento administrado mejora las lesiones cutáneas ${ }^{18,33}$. El receptor de estas quimiocinas, el CCR3, se expresa en las células tumorales $\mathrm{CD}_{30}+$, pero no se encuentra en las biopsias de piel en las fases tempranas de la micosis fungoide ${ }^{13}$, lo que sugiere que estas quimiocinas y sus receptores son importantes en los estadios avanzados del linfoma cutáneo de células $\mathrm{T}$ y favorecen un perfil Th2 que los excluye de la respuesta antitumoral del huésped ${ }^{34}$.

\section{Migración al ganglio en el síndrome de Sèzary: CCR7 y sus ligandos CCL19 y CCL21}

CCR7 es un receptor crítico para la migración de células dendríticas cutáneas maduras y linfocitos $\mathrm{T}$ a los ganglios linfáticos ${ }^{35}$. Se demostró que en el síndrome de Sèzary las células malignas de sangre periférica expresan altas concentraciones de CCR7, en comparación con controles sanos ${ }^{20,24}$. Las quimiocinas que se unen a dicho receptor, CCL19 y CCL21, aumentan la quimiotaxis de las células que expresan CCR7, en este caso, células del síndrome de Sèzary, y, al bloquear dicho receptor, 


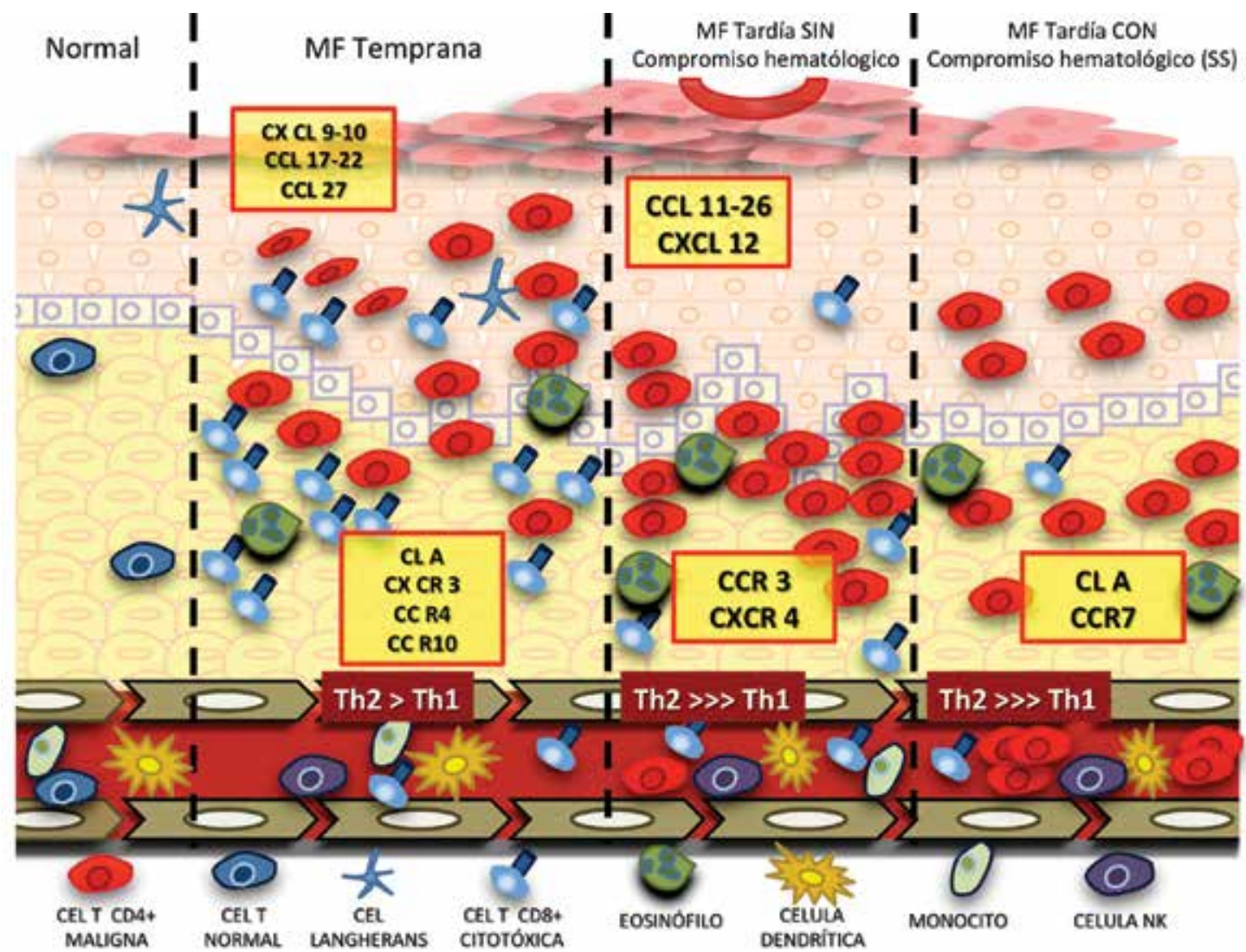

FIGURA 1. Micosis fungoide temprana. En fases iniciales de la micosis fungoide, el clon maligno expresa grandes cantidades de CXCR3, que favorecerían el epidermotropismo en respuesta al mayor gradiente de sus ligandos CXCL9 y CXCL10, producidos por los queratinocitos. CCL17 y CCL22 se encuentran en mayor concentración en los pacientes con linfoma cutáneo de células T; el clon maligno expresa su receptor, CCR4, lo que favorece su permanencia en piel. CCL27 se expresa exclusivamente en la piel y favorece el asentamiento del clon maligno que expresa CCR1o.

MICOSIS FUNGOIDE AVANZADA Y SÍNDROME DE SĖZARY. A medida que avanza la enfermedad hay mayor expresión en piel de CCL11 y CCL26 que favorecen una respuesta Th2, lo que disminuye la defensa antitumoral y la migración del clon maligno a ganglios y sangre periférica, ya que expresan el receptor CCR3. Las células de Sèzary expresan CXCR4, cuyo ligando CXCL12 favorece su permanencia en piel y la migración a otros tejidos. CCR7 se expresa en el clon maligno y es clave para la migración a los ganglios y otros tejidos por sangre periférica.

se disminuyó la migración a ganglios linfáticos mediada por estas quimiocinas ${ }^{36}$.

\section{Síndrome de Sèzary: CXCR4 y su ligando CXCL12}

Uno de los marcadores de las células del síndrome de Sèzary es la pérdida del CD26 en su membrana ${ }^{37}$. El CD26 es una peptidasa que escinde e inactiva CXCL12 ${ }^{38}$. Se estudió, entonces, si la CXCL12 y su receptor CXCR4 tenían alguna importancia en el síndrome de Sèzary. Se demostró que las células de Sèzary circulantes y las que infiltran la piel tiene gran expresión de CXCR4, y que migran más eficientemente en respuesta a CXCL12 que las células de controles sanos al ser estimuladas por diferentes concentraciones de la quimiocina ${ }^{39}$. La CXCL12 se expresa en células dendríticas, células epiteliales y linfocitos perivasculares, tanto en piel sana como en la afectada ${ }^{40}$.

\section{Quimiocinas y supervivencia celular}

En diferentes estudios se ha demostrado que la unión de las quimiocinas a sus receptores aumenta la supervivencia celular por medio de las vías de señalización de 


\begin{tabular}{|c|c|c|}
\hline $\begin{array}{l}\text { Receptor (expresado en linfocitos } \\
\text { T malignos y en otras células del } \\
\text { sistema inmunológico) }\end{array}$ & $\begin{array}{l}\text { Quimiocinas y células que las } \\
\text { producen }\end{array}$ & $\begin{array}{l}\text { Acciones fisiológicas y efectos en el linfoma } \\
\text { cutáneo de células T }\end{array}$ \\
\hline $\mathrm{CXCR}_{3}$ & CXCL9, CXCL10 & $\begin{array}{l}\text { Recluta linfocitos T Th1 a los sitios de } \\
\text { inflamación. }\end{array}$ \\
\hline (linfocitos T, células NK) & $\begin{array}{l}\text { Queratinocitos, células } \\
\text { endoteliales, fibroblastos }\end{array}$ & $\begin{array}{l}\text { CXCR3 está aumentado en las fases iniciales } \\
\text { de la micosis fungoide y podría mediar el } \\
\text { epidermotropismo en sus fases iniciales. }\end{array}$ \\
\hline CCR4 & CCL17, CCL22 & Asentamiento de los linfocitos en piel \\
\hline (linfocitos T) & $\begin{array}{l}\text { Queratinocitos, células } \\
\text { dendríticas, células endoteliales }\end{array}$ & $\begin{array}{l}\text { CCR4 está aumentado en las células tumorales, } \\
\text { y la expresión de CCL17 y CCL } 22 \text { se relaciona } \\
\text { con la actividad de la enfermedad. }\end{array}$ \\
\hline CCR10 & $\mathrm{CCL} 27$ & Regula el tráfico celular a la dermis. \\
\hline (linfocitos T, melanocitos) & $\begin{array}{l}\text { Queratinocitos y células } \\
\text { endoteliales }\end{array}$ & $\begin{array}{l}\text { Asentamiento en piel de células tumorales. } \\
\text { La CCL27 se relaciona con la actividad de la } \\
\text { enfermedad. }\end{array}$ \\
\hline $\mathrm{CCR}_{3}$ & CCL11, CCL26 & $\begin{array}{l}\text { Actividad quimiotáctica para eosinófilos y } \\
\text { células Th2 }\end{array}$ \\
\hline (leucocitos) & Fibroblastos, macrófagos & $\begin{array}{l}\text { En estadios avanzados del linfoma cutáneo } \\
\text { de células T, favorecen un ambiente Th2 que } \\
\text { disminuye la respuesta antitumoral. }\end{array}$ \\
\hline $\mathrm{CCR}_{7}$ & CCL19, CCL21 & Asentamiento de linfocitos T en ganglios \\
\hline (células dendríticas, linfocitos T) & $\begin{array}{l}\text { Células endoteliales de vasos } \\
\text { linfáticos y ganglios }\end{array}$ & Migración de células malignas a ganglios \\
\hline \multirow{2}{*}{$\begin{array}{l}\text { CXCR4 } \\
\text { (leucocitos, células dendríticas) }\end{array}$} & CXCL12 & Quimiotaxis de leucocitos \\
\hline & Células dendríticas, linfocitos & $\begin{array}{l}\text { Hay mayor expresión en células tumorales } \\
\text { y en células de Sèzary, lo que favorece el } \\
\text { asentamiento en piel. }\end{array}$ \\
\hline
\end{tabular}

TABLA 1. Receptores de quimiocinas y quimiocinas implicadas en la patogénesis de linfomas cutáneos de células T NK: natural killer

la proteína serina-treonina cinasa Akt (Protein Kinases $B$, PKB), la fosfatidil-inositol-3 cinasa (Phosphoinositide 3-Kinases, $\mathrm{PI} 3 \mathrm{~K}$ ) y la cinasa activada por mitógenos (Mitogen-activated protein kinases, MAPK ${ }^{41}$.

En estudios de células de melanoma se ha demostrado que la unión de las quimiocinas a su receptor acoplado a proteínas $G$, hace que la célula sea resistente a la apoptosis mediada por Fas y activa la fosforilación de
Akt mediada por $\mathrm{PI} 3 \mathrm{~K}$, ya que inhibidores de esta última disminuyen la supervivencia celular ${ }^{42}$. Faltan estudios que demuestren los mismos hallazgos en linfomas cutáneos de células T, pero, al tratarse de los mismos receptores y quimiocinas, es posible que se presenten efectos similares a los descritos. En la TABLA 1 y la Figura 1 se muestra el resumen de los aspectos más importantes de las quimiocinas en linfomas cutáneos de células T. 


\section{Quimiocinas como posibles blancos terapéuticos}

La micosis fungoide y el síndrome de Sèzary son enfermedades que, en fases avanzadas, pueden comprometer la vida del paciente por afectación de órganos sólidos, como el pulmón, el bazo y el hígado ${ }^{43}$. Una vez llegan a la fase IV, la supervivencia es de solo 13 meses y hay poca mejoría con los tratamientos convencionales a base de quimioterapia ${ }^{44,45}$. En la búsqueda de mejores tratamientos para mejorar la supervivencia, se han propuesto a las quimiocinas y sus receptores como posibles blancos terapéuticos. En estudios in vitro e in vivo en modelos en múridos, se ha demostrado que el anticuerpo monoclonal anti-CCR4 induce citotoxicidad dependiente de anticuerpos contra células de micosis fungoide y síndrome de Sèzary; además, hay una importante actividad antitumoral en modelos en múridos cuando se les administra dicho anticuerpo ${ }^{46,47}$.

También se han utilizado quimiocinas, como la CCL17 fusionadas con neurotoxinas o la exotoxina 38 de las pseudomonas, con lo cual se logró erradicar las células que expresaban su receptor después de la interiorización de la proteína de fusión ${ }^{48-50}$.

\section{Conclusión}

Las quimiocinas y sus receptores participan en el control de la migración, su presencia en la piel y la supervivencia de las células tumorales. Su expresión varía en los diferentes estadios del linfoma cutáneo de células T y difiere cuando se compara con la de controles sanos. En la actualidad se consideran importantes blancos terapéuticos y están bajo investigación con el propósito de ayudar al control de la progresión en las fases tempranas de la enfermedad y, en las fases tardías, para mejorar la supervivencia y la calidad de vida de los pacientes con linfoma cutáneo de células T.

\section{Referencias}

1. Laing KJ, Secombes CJ. Chemokines. Dev Comp Immunol. 2004;28: 443-60.

2. Sallusto F, Lenig D, Mackay CR, Lanzavecchia A. Flexible programs of chemokine receptor expression on human polarized T helper 1 and 2 lymphocytes. J Exp Med. 1998;187:875-83.

3. Murdoch C, Finn A. Chemokine receptors and their role in inflammation and infectious diseases. Blood. 2000;95:3032-43.

4. Groves FD, Linet MS, Travis LB, Devesa SS. Cancer surveillance series: Non-Hodgkin's lymphoma incidence by histologic subtype in the United States from 1978 through 1995. J Natl Cancer Inst. 2000;92:1240-51.
5. Willemze R, Jaffe ES, Burg G, Cerroni L, Berti E, Swerdlow SH, et al. WHO-EORTC classification for cutaneous lymphomas. Blood. 2005; 105:3768-85.

6. Olsen E, Vonderheid E, Pimpinelli N, Willemze R, Kim Y, Knobler $\mathrm{R}$, et al. Revisions to the staging and classification of mycosis fungoides and Sèzary syndrome: A proposal of the International Society for Cutaneous Lymphomas (ISCL) and the cutaneous lymphoma task force of the European Organization of Research and Treatment of Cancer (EORTC). Blood. 2007;110:1713-22.

7. Humme D, Lukowsky A, Sterry W. Diagnostic tools in mycosis fungoides. G Ital Dermatol Venereol. 2010;145:375-84.

8. Wood GS, Greenberg HL. Diagnosis, staging, and monitoring of cutaneous T-cell lymphoma. Dermatol Ther. 2003;16:269-75.

9. Momtaz P, Zippin JH. Cutaneous T-cell lymphoma: A review of current therapies and the future therapeutic implications of chemokine biology. J Drugs Dermatol. 2009;8:1142-9.

10. Hwang ST, Janik JE, Jaffe ES, Wilson WH. Mycosis fungoides and Sèzary syndrome. Lancet. 2008;371:945-57.

11. Loetscher M, Gerber B, Loetscher P, Jones SA, Piali L, Clark-Lewis I, et al. Chemokine receptor specific for IP10 and mig: Structure, function, and expression in activated T-lymphocytes. J Exp Med. 1996;184:963-9.

12. Lu D, Duvic M, Medeiros LJ, Luthra R, Dorfman DM, Jones D. The T-cell chemokine receptor CXCR3 is expressed highly in low-grade mycosis fungoides. Am J Clin Pathol. 2001;115:413-21.

13. Kallinich T, Muche JM, Qin S, Sterry W, Audring H, Kroczek RA. Chemokine receptor expression on neoplastic and reactive T cells in the skin at different stages of mycosis fungoides. J Invest Dermatol. 2003;121:1045-52.

14. Sarris AH, Esgleyes-Ribot T, Crow M, Broxmeyer HE, Karasavvas $\mathrm{N}$, Pugh W, et al. Cytokine loops involving interferon-gamma and IP-10, a cytokine chemotactic for CD4+ lymphocytes: An explanation for the epidermotropism of cutaneous T-cell lymphoma? Blood. 1995;86:651-8.

15. Tensen CP, Vermeer MH, van der Stoop PM, van Beek P, Scheper $\mathrm{RJ}$, Boorsma DM, et al. Epidermal interferon-gamma inducible protein-10 (IP-10) and monokine induced by gamma-interferon (Mig) but not IL-8 mRNA expression is associated with epidermotropism in cutaneous T cell lymphomas. J Invest Dermatol. 1998;111:222-6.

16. Sugaya M. Chemokines and cutaneous lymphoma. J Dermatol Sci. 2010;59:81-5.

17. Wu CS, Wang ST, Liao CY, Wu MT. Differential CCR4 expression and function in cutaneous T-cell lymphoma cell lines. Kaohsiung J Med Sci. 2008;24:577-90.

18. Yamaguchi T, Ohshima K, Karube K, Kawano R, Nakayama J, Suzumiya J, et al. Expression of chemokines and chemokine receptors in cutaneous $\mathrm{CD} 3 \mathrm{O}+$ lymphoproliferative disorders. $\mathrm{Br} \mathrm{J}$ Dermatol. 2006;154:904-9.

19. Kakinuma T, Sugaya M, Nakamura K, Kaneko F, Wakugawa M, Matsushima K, et al. Thymus and activation-regulated chemokine (TARC/CCL17) in mycosis fungoides: Serum TARC levels reflect the disease activity of mycosis fungoides. J Am Acad Dermatol. 2003;48:23-30.

20. Sokolowska-Wojdylo M, Wenzel J, Gaffal E, Lenz J, Speuser P, Erdmann S, et al. Circulating clonal CLA(+) and CD4(+) T cells in Sèzary syndrome express the skin-homing chemokine receptors CCR4 and CCR10 as well as the lymph node-homing chemokine receptor CCR7. Br J Dermatol. 2005;152:258-64. 
21. Richardson SK, Newton SB, Bach TL, Budgin JB, Benoit BM, Lin $\mathrm{JH}$, et al. Bexarotene blunts malignant T-cell chemotaxis in Sèzary syndrome: Reduction of chemokine receptor 4-positive lymphocytes and decreased chemotaxis to thymus and activation-regulated chemokine. Am J Hematol. 2007;82:792-7.

22. Homey B, Alenius H, Muller A, Soto H, Bowman EP, Yuan W, et al. CCL27-CCR1o interactions regulate $\mathrm{T}$ cell-mediated skin inflammation. Nat Med. 2002;8:157-65.

23. Morales J, Homey B, Vicari AP, Hudak S, Oldham E, Hedrick J, et al. CTACK, a skin-associated chemokine that preferentially attracts skin-homing memory T cells. Proc Natl Acad Sci. 1999;96:14470-5.

24. Capriotti E, Vonderheid EC, Thoburn CJ, Bright EC, Hess AD. Chemokine receptor expression by leukemic T cells of cutaneous T-cell lymphoma: Clinical and histopathological correlations. J Invest Dermatol. 2007;127:2882-92.

25. Notohamiprodjo M, Segerer S, Huss R, Hildebrandt B, Soler D, Djafarzadeh R, et al. CCR10 is expressed in cutaneous T-cell lymphoma. Int J Cancer. 2005;115:641-7.

26. Fujita Y, Abe R, Sasaki M, Honda A, Furuichi M, Asano Y, et al. Presence of circulating CCR10+ T cells and elevated serum CTACK/ CCL27 in the early stage of mycosis fungoides. Clin Cancer Res. 2006;12:2670-5.

27. Kagami S, Sugaya M, Minatani Y, Ohmatsu H, Kakinuma T, Fujita $\mathrm{H}$, et al. Elevated serum CTACK/CCL27 levels in CTCL. J Invest Dermatol. 2006;126:1189-91.

28. Masui Y, Sugaya M, Kagami S, Fujita H, Yano S, Nagao M, et al. Sèzary syndrome treated with narrowband ultraviolet B: Timecourse measurement of serum levels of CCL17/CCL27. Clin Exp Dermatol. 2007;32:57-9.

29. Hoeller C, Richardson SK, Ng LG, Valero T, Wysocka M, Rook AH, et al. In vivo imaging of cutaneous T-cell lymphoma migration to the skin. Cancer Res. 2009;69:2704-8.

30. Kitaura M, Nakajima T, Imai T, Harada S, Combadiere C, Tiffany $\mathrm{HL}$, et al. Molecular cloning of human eotaxin, an eosinophilselective CC chemokine, and identification of a specific eosinophil eotaxin receptor, CC chemokine receptor 3. J Biol Chem. 1996;271:7725-30.

31. Forssmann U, Uguccioni M, Loetscher P, Dahinden CA, Langen H, Thelen M, et al. Eotaxin-2, a novel CC chemokine that is selective for the chemokine receptor CCR3, and acts like eotaxin on human eosinophil and basophil leukocytes. J Exp Med. 1997;185:2171-6.

32. Uguccioni M, Mackay CR, Ochensberger B, Loetscher P, Rhis S, LaRosa GJ, et al. High expression of the chemokine receptor CCR3 in human blood basophils. Role in activation by eotaxin, MCP-4, and other chemokines. J Clin Invest. 1997;100:1137-43.

33. Miyagaki T, Sugaya M, Fujita H, Ohmatsu H, Kakinuma T, Kadono $\mathrm{T}$, et al. Eotaxins and $\mathrm{CCR}_{3}$ interaction regulates the Th2 environment of cutaneous T-cell lymphoma. J Invest Dermatol. 2010;130:2304-11.

34. Kleinhans M, Tun-Kyi A, Gilliet M, Kadin ME, Dummer R, Burg G, et al. Functional expression of the eotaxin receptor $\mathrm{CCR}_{3}$ in $\mathrm{CD}_{30}+$ cutaneous T-cell lymphoma. Blood. 2003;101:1487-93.

35. Forster R, Schubel A, Breitfeld D, Kremmer E, Renner-Muller I, Wolf $\mathrm{E}$, et al. $\mathrm{CCR} 7$ coordinates the primary immune response by establishing functional microenvironments in secondary lymphoid organs. Cell. 1999;99:23-33.
36. Picchio MC, Scala E, Pomponi D, Caprini E, Frontani M, Angelucci I, et al. CXCL13 is highly produced by Sèzary cells and enhances their migratory ability via a synergistic mechanism involving CCL19 and CCL21 chemokines. Cancer Res. 2008;68:7137-46.

37. Scala E, Narducci MG, Amerio P, Baliva G, Simoni R, Giovannetti $\mathrm{A}$, et al. $\mathrm{T}$ cell receptor-Vbeta analysis identifies a dominant CD6o+ CD26- CD49d- T cell clone in the peripheral blood of Sèzary syndrome patients. J Invest Dermatol. 2002;119:193-6.

38. Lambeir AM, Proost P, Durinx C, Bal G, Senten K, Augustyns K, et al. Kinetic investigation of chemokine truncation by $\mathrm{CD} 26 /$ dipeptidyl peptidase IV reveals a striking selectivity within the chemokine family. J Biol Chem. 2001;276:29839-45.

39. Narducci MG, Scala E, Bresin A, Caprini E, Picchio MC, Remotti D, et al. Skin homing of Sèzary cells involves SDF-1-CXCR4 signaling and down-regulation of CD26/dipeptidylpeptidase IV. Blood. 2006;107:1108-15.

40. Pablos JL, Amara A, Bouloc A, Santiago B, Caruz A, Galindo M, et al. Stromal-cell derived factor is expressed by dendritic cells and endothelium in human skin. Am J Pathol. 1999;155:1577-86.

41. Youn BS, Yu KY, Oh J, Lee J, Lee TH, Broxmeyer HE. Role of the CC chemokine receptor 9/TECK interaction in apoptosis. Apoptosis. 2002;7:271-6.

42. Murakami T, Cardones AR, Finkelstein SE, Restifo NP, Klaunberg BA, Nestle FO, et al. Immune evasion by murine melanoma mediated through CC chemokine receptor-10. J Exp Med. 2003;198:1337-47.

43. Kim YH, Hoppe RT. Mycosis fungoides and the Sèzary syndrome. Semin Oncol. 1999;26:276-89..

44. de Coninck EC, Kim YH, Varghese A, Hoppe RT. Clinical characteristics and outcome of patients with extracutaneous mycosis fungoides. J Clin Oncol. 2001;19:779-84.

45. Anadolu RY, Birol A, Sanli H, Erdem C, Tursen U. Mycosis fungoides and Sèzary syndrome: Therapeutic approach and outcome in 113 patients. Int J Dermatol. 2005;44:559-65.

46. Yano H, Ishida T, Inagaki A, Ishii T, Ding J, Kusumoto S, et al. Defucosylated anti CC chemokine receptor 4 monoclonal antibody combined with immunomodulatory cytokines: A novel immunotherapy for aggressive/refractory mycosis fungoides and Sèzary syndrome. Clin Cancer Res. 2007;13:6494-500.

47. Ishida T, Iida S, Akatsuka Y, Ishii T, Miyazaki M, Komatsu H, et al. The CC chemokine receptor 4 as a novel specific molecular target for immunotherapy in adult T-cell leukemia/lymphoma. Clin Cancer Res. 2004;10:7529-39.

48. Baatar D, Olkhanud P, Newton D, Sumitomo K, Biragyn A. CCR4expressing $\mathrm{T}$ cell tumors can be specifically controlled via delivery of toxins to chemokine receptors. J Immunol. 2007;179:1996-2004.

49. Wu XS, Lonsdorf AS, Hwang ST. Cutaneous T-cell lymphoma: Roles for chemokines and chemokine receptors. J Invest Dermatol. 2009;129:1115-9.

50. Junkins-Hopkins JM. Immunomodulatory therapy of cutaneous Tcell lymphoma: A multimodality approach in advanced disease. J Am Acad Dermatol. 2009;61:1056-8. 